\title{
Determining the Dirac CP Violation Phase and Neutrino Mass Hierarchy
}

\section{Zoran Bozidar Todorovic}

Faculty of Electrical Engineering, Department of Physics, University of Belgrade, Belgrade, Serbia

\author{
Email address: \\ tzoran221@gmail.com
}

\section{To cite this article:}

Zoran Bozidar Todorovic. Determining the Dirac CP Violation Phase and Neutrino Mass Hierarchy. American Journal of Modern Physics. Vol. 10, No. 4, 2021, pp. 60-70. doi: 10.11648/j.ajmp.20211004.11

Received: July 22, 2021; Accepted: August 2, 2021; Published: August 12, 2021

\begin{abstract}
There is still a problem in neutrino physics related to the configuration of neutrino masses: Are neutrinos arranged by masses following the Standard Model as three generations of fundamental particles, Gen III $>$ Gen II $>$ Gen I, thus forming a structural-normal hierarchy, or deviate from that principle? The biggest obstacle that is still present is the sign of the absolute value of the difference of the square of neutrino masses. It was avoided by applying the theory of neutrino oscillation probability for each structure of the neutrino mass hierarchy. With such theoretical approach the equation of motion was derived for each structure in which Dirac CP violation phase appeared as an unknown quantity. This enables direct calculation of the explicit value for the Dirac CP violation phase. Two examples were analyzed: The first example is devoted to the normal mass ordering and the second one is devoted to the inverted mass ordering. The data used for theoretical calculations presented in this paper are obtained on the basis of the latest reassessed data by processing the results of experimental measurements. On the basis of the performed calculations, normal mass ordering is unconditionally excluded as a potential option regarding the neutrino mass ordering in nature. On the basis of the derived equation of neutrino motion, a possible numerical value of the Dirac CP violation phase and Jarlskog invariant is found.
\end{abstract}

Keywords: Special Relativity, Leptons, Ordinary Neutrino, Neutrino Mass and Mixing, PMNS Matrix, Jarlskog Invariant, Dirac CP Phase

\section{Introduction}

The nature of neutrinos related to neutrino flavor oscillations was experimentally resolved [1-4], which made it clear that neutrinos could possess mass. The entire theory on the oscillations of neutrino flavor states is based on the application of the unitary PMNS mixing matrix containing parameters which connect flavor eigenstates with mass eigenstates. Those parameters provide mismatches between flavor states and mass eigenstates that are necessary for establishing oscillations between certain neutrino flavor states.

In the standard scenario, the three neutrinos $v_{1}, v_{2}, v_{3}$ are known to have relative masses measured as $\Delta m_{21}^{2}=m_{2}^{2}-m_{1}^{2}$ and $\Delta m_{31}^{2}=\left|m_{3}^{2}-m_{1}^{2}\right|$. In neutrino physics, the sign of $\Delta m_{31}^{2}$ is still debatable and unspecified as it has not been measured yet, and that allows two different configurations for the masses: either $m_{1}<m_{2}<m_{3}$ (normal mass ordering) or $m_{3}<m_{1}<m_{2}$. (inverted mass ordering). The dilemmas in neutrino physics related to the sign of $\Delta m_{31}^{2}$ still present an obstacle for defining the neutrino mass ordering. However, we will see that the precise definition of the mass splittings between neutrino mass eigenstates, which is done in the latest analysis of experimental data [10], can be of crucial importance for defining the nature of neutrino mass hierarchy. The Standard Model has three generations of fundamental matter particles. Three generations of the quark and charged lepton mass show a hierarchical structure: Gen III $>$ Gen II $>$ Gen I. Owing to that, there is a belief and it is considered that neutrinos may follow such hierarchical structure. Thus, a justified question is raised: Does neutrino mass show the same structure?

And, owing to that and in relation to that, it should be noted that there are more open questions in neutrino physics that are waiting to be answered [1-6]: 
Are squared neutrino masses ordered normally $m_{1}^{2}<m_{2}^{2}<m_{3}^{2}$ or are they inverted $m_{3}^{2}<m_{1}^{2}<m_{2}^{2}$ ?

What is the numerical value of the Dirac CP violation phase?

What is the numerical value of the Jarlskog invariant?

What is the lowest neutrino mass? What is the absolute mass of a neutrino?

Do neutrinos and antineutrinos behave differently? Is a neutrino its own antiparticle?

In the researches presented in this paper, we will deal with the matters related to the listed points 1,2 and 3 .

\section{Defining Basic Relations in Neutrino Physics}

Let the wavelengths of oscillations be denoted by $L_{i j}(i, j=1,2,3)$, linking them to the differences of the appropriate phases $\varphi_{i j}(i, j=1,2,3)$, and then relations for the processes of disappearances can be written as follows:

Normal mass ordering, $m_{1}<m_{2}<m_{3}$

$$
\begin{aligned}
& \left(v_{e} \rightarrow v_{\mu} \rightarrow v_{e}\right) \rightarrow \varphi_{1}-\varphi_{2}=\varphi_{12}=\frac{L_{12}}{\hbar}\left(p_{1}-p_{2}\right)=2 \pi \\
& \left(v_{e} \rightarrow v_{\tau} \rightarrow v_{e}\right) \rightarrow \varphi_{1}-\varphi_{3}=\varphi_{13}=\frac{L_{13}}{\hbar}\left(p_{1}-p_{3}\right)=2 \pi, \\
& \left(v_{\mu} \rightarrow v_{\tau} \rightarrow v_{\mu}\right) \rightarrow \varphi_{2}-\varphi_{3}=\varphi_{23}=\frac{L_{23}}{\hbar}\left(p_{2}-p_{3}\right)=2 \pi
\end{aligned}
$$

The first relation presents the process of oscillation of the electron neutrino through muon neutrino when one full oscillation is performed $L_{12}$.

The second relation presents the process of oscillation of

$$
\begin{aligned}
& \varphi_{12}=\frac{L_{12}}{\hbar}\left(p_{1}-p_{2}\right)=\frac{L_{12}}{\hbar}\left[E / c\left(1-\delta_{1}\right)-E / c\left(1-\delta_{2}\right)\right]=\frac{L_{12}}{\hbar}\left[E / c\left(\delta_{2}-\delta_{1}\right)\right] \\
& =\frac{L_{12}}{\hbar} \frac{E}{c}\left(\frac{m_{2}^{2} c^{4}}{2 E^{2}}-\frac{m_{1}^{2} c^{4}}{2 E^{2}}\right)=\frac{L_{12} c^{3}}{2 \hbar E}\left(m_{2}^{2}-m_{1}^{2}\right)=\frac{L_{12} c^{3}}{2 \hbar E} \Delta m_{21}^{2}=2 \pi \\
& m_{3}>m_{2}>m_{1} ; \delta_{1}=\frac{m_{1}^{2} c^{4}}{2 E^{2}}<<1, \delta_{2}=\frac{m_{2}^{2} c^{4}}{2 E^{2}}<<1, \delta_{3}=\frac{m_{3}^{2} c^{4}}{2 E^{2}} \ll<1 . \\
& \varphi_{23}=\frac{L_{12}}{\hbar}\left(p_{2}-p_{3}\right)=\frac{L_{12}}{\hbar}\left[E / c\left(\delta_{3}-\delta_{2}\right)\right]=\frac{L_{12} c^{3}}{2 \hbar E} \Delta m_{23}^{2}=2 \pi \frac{\Delta m_{32}^{2}}{\Delta m_{21}^{2}} . \\
& \varphi_{13}=\frac{L_{12}}{\hbar}\left(p_{1}-p_{3}\right)=\frac{L_{12}}{\hbar}\left[E / c\left(\delta_{3}-\delta_{1}\right)\right]=\frac{L_{12} c^{3}}{2 \hbar E} \Delta m_{31}^{2}=2 \pi \frac{\Delta m_{31}^{2}}{\Delta m_{21}^{2}}
\end{aligned}
$$

Where $\mathrm{c}$ is the speed of light, and $\hbar=h / 2 \pi$. And one more equation can be written:

$$
\Delta m_{21}^{2}+\Delta m_{32}^{2}=\Delta m_{31}^{2}
$$

Inverted mass ordering, $m_{3}<m_{1}<m_{2}$

$$
\begin{aligned}
& \left(v_{e} \rightarrow v_{\mu} \rightarrow v_{e}\right) \rightarrow \varphi_{1}-\varphi_{2}=\varphi_{12}=\frac{L_{12}}{\hbar}\left(p_{1}-p_{2}\right)=2 \pi \\
& \left(v_{e} \rightarrow v_{\tau} \rightarrow v_{e}\right) \rightarrow \varphi_{3}-\varphi_{1}=\varphi_{31}=\frac{L_{31}}{\hbar}\left(p_{3}-p_{1}\right)=2 \pi, \\
& \left(v_{\mu} \rightarrow v_{\tau} \rightarrow v_{\mu}\right) \rightarrow \varphi_{3}-\varphi_{2}=\varphi_{32}=\frac{L_{32}}{\hbar}\left(p_{3}-p_{2}\right)=2 \pi .
\end{aligned}
$$

Where it can be seen that the product of wavelengths of momentums equals the Planck constant.

In further research, we form the differences of phases of mass eigenstates on the distance $X=L_{12}$ from the source of the neutrino beam, moving through a physical vacuum, and they can be described by following equations:

\footnotetext{
Inverted mass ordering, $m_{3}<m_{1}<m_{2}$
}

The first relation describes the process of oscillations of the electron neutrino through muon neutrino when one full 
oscillation is performed $L_{12}$.

The second relation presents the process of oscillations of the electron neutrino through tau neutrino when one full oscillation is performed $L_{31}$.

The third relation presents the process of oscillations of the muon neutrino through tau neutrino when one full oscillation is performed $L_{32}$.

The momentum $p_{1}$ is linked to the mass eigenstate $m_{1}$, the momentum $p_{2}$ is linked to the mass eigenstate $m_{2}$, the momentum $p_{3}$ is linked to the mass eigenstate $m_{3}$. The equations signify that the product of wavelengths of neutrino oscillations and corresponding differences of the momentums equals the Planck constant.

From the relations (10), the following equations directly follow:

$$
\begin{aligned}
& L_{12}\left(p_{1}-p_{2}\right)=h \\
& L_{31}\left(p_{3}-p_{1}\right)=h \\
& \varphi_{12}=\frac{L_{12}}{\hbar}\left(p_{1}-p_{2}\right)=\frac{L_{12}}{\hbar}\left[E / c\left(\delta_{2}-\delta_{1}\right)\right]=\frac{L_{12} c^{3}}{2 \hbar E} \Delta m_{21}^{2}=2 \pi \\
& m_{3}<m_{1}<m_{2} ; \delta_{1}=\frac{m_{1}^{2} c^{4}}{2 E^{2}}<<1, \delta_{2}=\frac{m_{2}^{2} c^{4}}{2 E^{2}}<<1, \delta_{3}=\frac{m_{3}^{2} c^{4}}{2 E^{2}}<<1 . \\
& \varphi_{32}=\frac{L_{12}}{\hbar}\left(p_{3}-p_{2}\right)=\frac{L_{12}}{\hbar}\left[E / c\left(\delta_{2}-\delta_{3}\right)\right]=\frac{L_{12} c^{3}}{2 \hbar E} \Delta m_{23}^{2}=2 \pi \frac{\Delta m_{23}^{2}}{\Delta m_{21}^{2}} \\
& \varphi_{31}=\frac{L_{12}}{\hbar}\left(p_{3}-p_{1}\right)=\frac{L_{12}}{\hbar}\left[E / c\left(\delta_{1}-\delta_{3}\right)\right]=\frac{L_{12} c^{3}}{2 \hbar E} \Delta m_{13}^{2}=2 \pi \frac{\Delta m_{13}^{2}}{\Delta m_{21}^{2}}
\end{aligned}
$$

Where $\mathrm{c}$ is the speed of light, and $\hbar=h / 2 \pi$. And one more equation can be written:

$$
\Delta m_{21}^{2}+\Delta m_{13}^{2}=\Delta m_{23}^{2}
$$

\section{Application of the $U_{P M N S}^{P D G}$ Mixing Matrix}

In the processes known as neutrino flavor oscillations, the Dirac CP violation phase $\delta_{C P}$ is unequivocally singled out as the cause of those oscillations in the propagation of the neutrino beam through the physical vacuum. For that reason, there arises the question of writing the equation of motion in which $\delta_{C P}$ would appear as an unknown quantity. On the basis of that equation, it would be possible to determine that unknown quantity. So far, there appears to be only one way to derive equations of motion for a neutrino beam, and it is related to the use of the equations of the neutrino oscillations probabilities. The procedure for deriving those equations is given here.

\subsection{The Case of Normal Hierarchy of Neutrino Mass (NO)}

In this case, the matrix $U_{P M N S}^{P D G}$ is used $[5,8,9,11,12]$

$$
\begin{aligned}
& U_{P M N S}^{P D G}=\left(\begin{array}{ccc}
U_{e 1} & U_{e 2} & U_{e 3} \\
U_{\mu 1} & U_{\mu 2} & U_{\mu 3} \\
U_{\tau 1} & U_{\tau 2} & U_{\tau 3}
\end{array}\right)=\left(\begin{array}{ccc}
c_{12} c_{13} & s_{12} c_{13} & s_{13} e^{-i \delta_{C P}} \\
-s_{12} c_{23}-c_{12} s_{23} s_{13} e^{i \delta_{C P}} & c_{12} c_{23}-s_{12} s_{23} s_{13} e^{i \delta_{C P}} & c_{13} s_{23} \\
s_{12} s_{23}-c_{12} c_{23} s_{13} e^{i \delta_{C P}} & -c_{12} s_{23}-s_{12} c_{23} s_{13} e^{i \delta_{C P}} & c_{13} c_{23}
\end{array}\right) \\
& =\left(\begin{array}{ccc}
U_{e 1} & U_{e 2} & J e^{-i \delta_{C P}} \\
-A-B e^{i \delta_{C P}} & C-D e^{i \delta_{C P}} & U_{\mu 3} \\
E-F e^{i \delta_{C P}} & -G-H e^{i \delta_{C P}} & U_{\tau 3}
\end{array}\right)
\end{aligned}
$$

where the mixing angles are taken into consideration [10]: 


$$
\begin{aligned}
& \theta_{12}=34.3 \pm 1^{0}, \theta_{23}=49.26 \pm 0.79^{0}, \theta_{13}=8.53_{-0.53}^{+0.130}, \\
& c_{i j}=\cos \theta_{i j}, s_{i j}=\sin \theta_{i j} ; i, j=1,2,3 .
\end{aligned}
$$

In order to obtain an explicit numerical value of $\delta_{C P}$, the following unconditional rule will be applied: The sum of the probabilities of three neutrino oscillations during the transition $v_{e} \rightarrow v_{\mu}, v_{e} \rightarrow v_{\tau}, v_{e} \rightarrow v_{e}$, at a distance from the source equal to the entire wavelength of oscillations in the value of $X=L_{12}$, during the process of the disappearance in transition $v_{e} \rightarrow v_{\mu} \rightarrow v_{e}$, in the propagation of the neutrino beam through vacuum (as it can be seen, the matter effect is excluded in these considerations), is equal to one.

P. F. de Salas [10] provides the following data as well:

$$
\Delta m_{21}^{2}=7.50_{-0.20}^{+0.22} \times 10^{-5} \mathrm{eV}^{2}, \Delta m_{31}^{2}=2.55_{-0.03}^{+0.02} \times 10^{-3} \mathrm{eV}^{2}, \Delta m_{32}^{2}=2.475 \times 10^{-3} \mathrm{eV}^{2}
$$

Taking into consideration the central values from the data (20), we get:

$$
\begin{aligned}
& \frac{\Delta m_{31}^{2}}{\Delta m_{21}^{2}}=\frac{0.00255}{0.0000750}=34.0, \frac{\Delta m_{32}^{2}}{\Delta m_{21}^{2}}=\frac{0.002475}{0.0000750}=33.0, \\
& V=\sin \left(2 \pi \frac{\Delta m_{31}^{2}}{\Delta m_{21}^{2}}\right)=0, V=\sin \left(2 \pi \frac{\Delta m_{32}^{2}}{\Delta m_{21}^{2}}\right)=0 \\
& W=\sin ^{2}\left(\pi \frac{\Delta m_{31}^{2}}{\Delta m_{21}^{2}}\right)=0, W=\sin ^{2}\left(\pi \frac{\Delta m_{32}^{2}}{\Delta m_{21}^{2}}\right)=0 .
\end{aligned}
$$

In our considerations, we will use the general formula for neutrino oscillations given in Ref $[8,9]$ :

$$
P\left(v_{\alpha} \rightarrow v_{\beta}\right)=\delta_{\alpha \beta}-4 \sum_{i,<j} R\left(U_{\alpha i} U_{\beta i}^{*} U_{\alpha j}^{*} U_{\beta j}\right) \sin ^{2} \frac{\Delta m_{j i}^{2} L c^{3}}{4 E \hbar}+2 \sum_{i,<j} \operatorname{Im}\left(U_{\alpha i} U_{\beta i}^{*} U_{\alpha j}^{*} U_{\beta j}\right) \sin \frac{\Delta m_{j i}^{2} L c^{3}}{2 E \hbar} ; i, j=1,2,3
$$

The transition is analysed: $v_{e} \rightarrow v_{\mu}, v_{e} \rightarrow v_{\tau}, v_{e} \rightarrow v_{e}$

On the basis of formulae (22), the total probability of neutrino oscillations is shown through the equation

$$
\begin{aligned}
& P\left(v_{e} \rightarrow v_{\mu}\right)+P\left(v_{e} \rightarrow v_{\tau}\right)+P\left(v_{e} \rightarrow v_{e}\right) \\
& =1-4 R\left\{U_{e 1} U_{\mu 1}^{*} U_{e 2}^{*} U_{\mu 2} \sin ^{2} \pi \frac{\Delta m_{21}^{2}}{\Delta m_{21}^{2}}\right\}+2 \operatorname{Im}\left\{U_{e 1} U_{\mu 1}^{*} U_{e 2}^{*} U_{\mu 2} \sin 2 \pi \frac{\Delta m_{21}^{2}}{\Delta m_{21}^{2}}\right\} \\
& -4 R\left\{U_{e 1} U_{\mu 1}^{*} U_{e 3}^{*} U_{\mu 3} \sin ^{2} \pi \frac{\Delta m_{31}^{2}}{\Delta m_{21}^{2}}\right\}+2 \operatorname{Im}\left\{U_{e 1} U_{\mu 1}^{*} U_{e 3}^{*} U_{\mu 3} \sin 2 \pi \frac{\Delta m_{31}^{2}}{\Delta m_{21}^{2}}\right\} \\
& -4 R\left\{U_{e 2} U_{\mu 2}^{*} U_{e 3}^{*} U_{\mu 3} \sin ^{2} \pi \frac{\Delta m_{32}^{2}}{\Delta m_{21}^{2}}\right\}+2 \operatorname{Im}\left\{U_{e 2} U_{\mu 2}^{*} U_{e 3}^{*} U_{\mu 3} \sin 2 \pi \frac{\Delta m_{32}^{2}}{\Delta m_{21}^{2}}\right\} \\
& -4 R\left\{U_{e 1} U_{\tau 1}^{*} U_{e 2}^{*} U_{\tau 2} \sin ^{2} \pi \frac{\Delta m_{21}^{2}}{\Delta m_{21}^{2}}\right\}+2 \operatorname{Im}\left\{U_{e 1} U_{\tau 1}^{*} U_{e 2}^{*} U_{\tau 2} \sin 2 \pi \frac{\Delta m_{21}^{2}}{\Delta m_{21}^{2}}\right\} \\
& -4 R\left\{U_{e 1} U_{\tau 1}^{*} U_{e 3}^{*} U_{\tau 3} \sin ^{2} \pi \frac{\Delta m_{31}^{2}}{\Delta m_{21}^{2}}\right\}+2 \operatorname{Im}\left\{U_{e 1} U_{\tau 1}^{*} U_{e 3}^{*} U_{\tau 3} \sin 2 \pi \frac{\Delta m_{31}^{2}}{\Delta m_{21}^{2}}\right\} \\
& -4 R\left\{U_{e 2} U_{\tau 2}^{*} U_{e 3}^{*} U_{\tau 3} \sin ^{2} \pi \frac{\Delta m_{32}^{2}}{\Delta m_{21}^{2}}\right\}+2 \operatorname{Im}\left\{U_{e 2} U_{\tau 2}^{*} U_{e 3}^{*} U_{\tau 3} \sin 2 \pi \frac{\Delta m_{32}^{2}}{\Delta m_{21}^{2}}\right\} \\
& -4\left|U_{e 1}\right|^{2}\left|U_{e 2}\right|^{2} \sin ^{2} \pi \frac{\Delta m_{21}^{2}}{\Delta m_{21}^{2}}-4\left|U_{e 1}\right|^{2}\left|U_{e 3}\right|^{2} \sin ^{2} \pi \frac{\Delta m_{31}^{2}}{\Delta m_{21}^{2}} \\
& -4\left|U_{e 2}\right|^{2}\left|U_{e 3}\right|^{2} \sin ^{2} \pi \frac{\Delta m_{32}^{2}}{\Delta m_{21}^{2}}=1
\end{aligned}
$$

And, from the equation (23), the equation of neutrino motion is formed with a condition that the travelled distance of the neutrino beam, moving through a vacuum from the source, equals the neutrino wavelength $X=L_{12}$. So, it can be written as 


$$
\begin{aligned}
& -4 R\left\{U_{e 1} U_{\mu 1}^{*} U_{e 2}^{*} U_{\mu 2} \sin ^{2} \pi \frac{\Delta m_{21}^{2}}{\Delta m_{21}^{2}}\right\}+2 \operatorname{Im}\left\{U_{e 1} U_{\mu 1}^{*} U_{e 2}^{*} U_{\mu 2} \sin 2 \pi \frac{\Delta m_{21}^{2}}{\Delta m_{21}^{2}}\right\} \\
& -4 R\left\{U_{e 1} U_{\mu 1}^{*} U_{e 3}^{*} U_{\mu 3} \sin ^{2} \pi \frac{\Delta m_{31}^{2}}{\Delta m_{21}^{2}}\right\}+2 \operatorname{Im}\left\{U_{e 1} U_{\mu 1}^{*} U_{e 3}^{*} U_{\mu 3} \sin 2 \pi \frac{\Delta m_{31}^{2}}{\Delta m_{21}^{2}}\right\} \\
& -4 R\left\{U_{e 2} U_{\mu 2}^{*} U_{e 3}^{*} U_{\mu 3} \sin ^{2} \pi \frac{\Delta m_{32}^{2}}{\Delta m_{21}^{2}}\right\}+2 \operatorname{Im}\left\{U_{e 2} U_{\mu 2}^{*} U_{e 3}^{*} U_{\mu 3} \sin 2 \pi \frac{\Delta m_{32}^{2}}{\Delta m_{21}^{2}}\right\} \\
& -4 R\left\{U_{e 1} U_{\tau 1}^{*} U_{e 2}^{*} U_{\tau 2} \sin ^{2} \pi \frac{\Delta m_{21}^{2}}{\Delta m_{21}^{2}}\right\}+2 \operatorname{Im}\left\{U_{e 1} U_{\tau 1}^{*} U_{e 2}^{*} U_{\tau 2} \sin 2 \pi \frac{\Delta m_{21}^{2}}{\Delta m_{21}^{2}}\right\} \\
& -4 R\left\{U_{e 1} U_{\tau 1}^{*} U_{e 3}^{*} U_{\tau 3} \sin ^{2} \pi \frac{\Delta m_{31}^{2}}{\Delta m_{21}^{2}}\right\}+2 \operatorname{Im}\left\{U_{e 1} U_{\tau 1}^{*} U_{e 3}^{*} U_{\tau 3} \sin 2 \pi \frac{\Delta m_{31}^{2}}{\Delta m_{21}^{2}}\right\} \\
& -4 R\left\{U_{e 2} U_{\tau 2}^{*} U_{e 3}^{*} U_{\tau 3} \sin ^{2} \pi \frac{\Delta m_{32}^{2}}{\Delta m_{21}^{2}}\right\}+2 \operatorname{Im}\left\{U_{e 2} U_{\tau 2}^{*} U_{e 3}^{*} U_{\tau 3} \sin 2 \pi \frac{\Delta m_{32}^{2}}{\Delta m_{21}^{2}}\right\} \\
& -4\left|U_{e 1}\right|^{2}\left|U_{e 2}\right|^{2} \sin ^{2} \pi \frac{\Delta m_{21}^{2}}{\Delta m_{21}^{2}}-4\left|U_{e 1}\right|^{2}\left|U_{e 3}\right|^{2} \sin ^{2} \pi \frac{\Delta m_{31}^{2}}{\Delta m_{21}^{2}} \\
& -4\left|U_{e 2}\right|^{2}\left|U_{e 3}\right|^{2} \sin ^{2} \pi \frac{\Delta m_{32}^{2}}{\Delta m_{21}^{2}}=0
\end{aligned}
$$

Inserting data (21), it can be seen that all members of the equation (24), with no exception, become equal to zero, and that it is always the case regardless of the values of the elements of PMNS mixing matrix (19) and their connections with the Dirac $\mathrm{CP}$ violation phase.

The complex structure of equations (24) is reduced to an extremely simple form:

$$
\left(4 W \cos \delta_{C P}-2 V \sin \delta_{C P}\right) * 0=\left(4 \cos \delta_{C P} * 0-2 \sin \delta_{C P} * 0\right) * 0=0
$$

Every member of the equation (25) equals zero. That means that $\mathrm{CP}$ phases can have any arbitrarily taken value from the interval $(0,2 \pi)$. This result can be considered an irrefutable proof that, in nature, neutrinos, in the hierarchy of the mass eigenstates, do not belong to the normal mass ordering, $m_{1}<m_{2}<m_{3}$. From this, the only conclusion that follows is that, in nature, the neutrino mass eigenstates belong to the inverted mass ordering, $m_{3}<m_{1}<m_{2}$ to which we shall pay further attention.

\subsection{The Case of Inverted Hierarchy of Neutrino Masses (IO)}

And, in this case, the matrix $U_{P M N S}^{P D G}$ is used $[5,8,9,11,12]$

$$
\begin{aligned}
& U_{P M N S}^{P D G}=\left(\begin{array}{ccc}
U_{e 1} & U_{e 2} & U_{e 3} \\
U_{\mu 1} & U_{\mu 2} & U_{\mu 3} \\
U_{\tau 1} & U_{\tau 2} & U_{\tau 3}
\end{array}\right) \\
& =\left(\begin{array}{ccc}
c_{12} c_{13} & s_{12} c_{13} & s_{13} e^{-i \delta_{C P}} \\
-s_{12} c_{23}-c_{12} s_{23} s_{13} e^{i \delta_{C P}} & c_{12} c_{23}-s_{12} s_{23} s_{13} e^{i \delta_{C P}} & c_{13} s_{23} \\
s_{12} s_{23}-c_{12} c_{23} s_{13} e^{i \delta_{C P}} & -c_{12} s_{23}-s_{12} c_{23} s_{13} e^{i \delta_{C P}} & c_{13} c_{23}
\end{array}\right) \\
& =\left(\begin{array}{ccc}
U_{e 1} & U_{e 2} & J e^{-i \delta_{C P}} \\
-A-B e^{i \delta_{C P}} & C-D e^{i \delta_{C P}} & U_{\mu 3} \\
E-F e^{i \delta_{C P}} & -G-H e^{i \delta_{C P}} & U_{\tau 3}
\end{array}\right)
\end{aligned}
$$

For determining values of PMNS matrix elements, as well as for defining elements of the motion equation, the data given in the Ref. [10] are used: 


$$
\begin{gathered}
\theta_{12}=34.3 \pm 1^{0}, \theta_{23}=49.46 \pm 0.79^{0}, \theta_{13}=8.58_{-0.53}^{+0.130}, c_{i j}=\cos \theta_{i j}, s_{i j}=\sin \theta_{i j} ; \\
i, j=1,2,3 . . \\
\Delta m_{21}^{2}=7.50_{-0.20}^{+0.22} \times 10^{-5} \mathrm{eV}^{2}, \Delta m_{13}^{2}=2.45_{-0.03}^{+0.02} \times 10^{-3} \mathrm{eV}^{2}, \Delta m_{23}^{2}=2.525 \times 10^{-3} \mathrm{eV}^{2}
\end{gathered}
$$

To obtain an explicit numerical value of $\delta_{C P}$, the following unconditional rule will be applied: The sum of the probabilities of three neutrino oscillations during the transition $v_{e} \rightarrow v_{\mu}, v_{e} \rightarrow v_{\tau}, v_{e} \rightarrow v_{e}$, at a distance from the source equal to the wavelength of oscillations in the value of $X=L_{12}$. For this transition, total oscillation probabilities equal one:

$$
\begin{aligned}
& P\left(v_{e} \rightarrow v_{\mu}\right)+P\left(v_{e} \rightarrow v_{\tau}\right)+P\left(v_{e} \rightarrow v_{e}\right) \\
& =1-4 R\left\{U_{e 1} U_{\mu 1}^{*} U_{e 2}^{*} U_{\mu 2} \sin ^{2} \pi \frac{\Delta m_{21}^{2}}{\Delta m_{21}^{2}}\right\}+2 \operatorname{Im}\left\{U_{e 1} U_{\mu 1}^{*} U_{e 2}^{*} U_{\mu 2} \sin 2 \pi \frac{\Delta m_{21}^{2}}{\Delta m_{21}^{2}}\right\} \\
& -4 R\left\{U_{e 1} U_{\mu 1}^{*} U_{e 3}^{*} U_{\mu 3} \sin ^{2} \pi \frac{\Delta m_{31}^{2}}{\Delta m_{21}^{2}}\right\}+2 \operatorname{Im}\left\{U_{e 1} U_{\mu 1}^{*} U_{e 3}^{*} U_{\mu 3} \sin 2 \pi \frac{\Delta m_{31}^{2}}{\Delta m_{21}^{2}}\right\} \\
& -4 R\left\{U_{e 2} U_{\mu 2}^{*} U_{e 3}^{*} U_{\mu 3} \sin ^{2} \pi \frac{\Delta m_{32}^{2}}{\Delta m_{21}^{2}}\right\}+2 \operatorname{Im}\left\{U_{e 2} U_{\mu 2}^{*} U_{e 3}^{*} U_{\mu 3} \sin 2 \pi \frac{\Delta m_{32}^{2}}{\Delta m_{21}^{2}}\right\} \\
& -4 R\left\{U_{e 1} U_{\tau 1}^{*} U_{e 2}^{*} U_{\tau 2} \sin ^{2} \pi \frac{\Delta m_{21}^{2}}{\Delta m_{21}^{2}}\right\}+2 \operatorname{Im}\left\{U_{e 1} U_{\tau 1}^{*} U_{e 2}^{*} U_{\tau 2} \sin 2 \pi \frac{\Delta m_{21}^{2}}{\Delta m_{21}^{2}}\right\} \\
& -4 R\left\{U_{e 1} U_{\tau 1}^{*} U_{e 3}^{*} U_{\tau 3} \sin ^{2} \pi \frac{\Delta m_{31}^{2}}{\Delta m_{21}^{2}}\right\}+2 \operatorname{Im}\left\{U_{e 1} U_{\tau 1}^{*} U_{e 3}^{*} U_{\tau 3} \sin 2 \pi \frac{\Delta m_{31}^{2}}{\Delta m_{21}^{2}}\right\} \\
& -4 R\left\{U_{e 2} U_{\tau 2}^{*} U_{e 3}^{*} U_{\tau 3} \sin ^{2} \pi \frac{\Delta m_{32}^{2}}{\Delta m_{21}^{2}}\right\}+2 \operatorname{Im}\left\{U_{e 2} U_{\tau 2}^{*} U_{e 3}^{*} U_{\tau 3} \sin 2 \pi \frac{\Delta m_{32}^{2}}{\Delta m_{21}^{2}}\right\} \\
& -4\left|U_{e 1}\right|^{2}\left|U_{e 2}\right|^{2} \sin ^{2} \pi \frac{\Delta m_{21}^{2}}{\Delta m_{21}^{2}}-4\left|U_{e 1}\right|^{2}\left|U_{e 3}\right|^{2} \sin ^{2} \pi \frac{\Delta m_{31}^{2}}{\Delta m_{21}^{2}}-4\left|U_{e 2}\right|^{2}\left|U_{e 3}\right|^{2} \sin ^{2} \pi \frac{\Delta m_{32}^{2}}{\Delta m_{21}^{2}}=1
\end{aligned}
$$

From the equation (28), the following form of the motion equation ensues:

$$
\begin{aligned}
& -4 R\left\{U_{e 1} U_{\mu 1}^{*} U_{e 2}^{*} U_{\mu 2} \sin ^{2} \pi \frac{\Delta m_{21}^{2}}{\Delta m_{21}^{2}}\right\}+2 \operatorname{Im}\left\{U_{e 1} U_{\mu 1}^{*} U_{e 2}^{*} U_{\mu 2} \sin 2 \pi \frac{\Delta m_{21}^{2}}{\Delta m_{21}^{2}}\right\} \\
& -4 R\left\{U_{e 1} U_{\mu 1}^{*} U_{e 3}^{*} U_{\mu 3} \sin ^{2} \pi \frac{\Delta m_{31}^{2}}{\Delta m_{21}^{2}}\right\}+2 \operatorname{Im}\left\{U_{e 1} U_{\mu 1}^{*} U_{e 3}^{*} U_{\mu 3} \sin 2 \pi \frac{\Delta m_{31}^{2}}{\Delta m_{21}^{2}}\right\} \\
& -4 R\left\{U_{e 2} U_{\mu 2}^{*} U_{e 3}^{*} U_{\mu 3} \sin ^{2} \pi \frac{\Delta m_{32}^{2}}{\Delta m_{21}^{2}}\right\}+2 \operatorname{Im}\left\{U_{e 2} U_{\mu 2}^{*} U_{e 3}^{*} U_{\mu 3} \sin 2 \pi \frac{\Delta m_{32}^{2}}{\Delta m_{21}^{2}}\right\} \\
& -4 R\left\{U_{e 1} U_{\tau 1}^{*} U_{e 2}^{*} U_{\tau 2} \sin ^{2} \pi \frac{\Delta m_{21}^{2}}{\Delta m_{21}^{2}}\right\}+2 \operatorname{Im}\left\{U_{e 1} U_{\tau 1}^{*} U_{e 2}^{*} U_{\tau 2} \sin 2 \pi \frac{\Delta m_{21}^{2}}{\Delta m_{21}^{2}}\right\} \\
& -4 R\left\{U_{e 1} U_{\tau 1}^{*} U_{e 3}^{*} U_{\tau 3} \sin ^{2} \pi \frac{\Delta m_{31}^{2}}{\Delta m_{21}^{2}}\right\}+2 \operatorname{Im}\left\{U_{e 1} U_{\tau 1}^{*} U_{e 3}^{*} U_{\tau 3} \sin 2 \pi \frac{\Delta m_{31}^{2}}{\Delta m_{21}^{2}}\right\} \\
& -4 R\left\{U_{e 2} U_{\tau 2}^{*} U_{e 3}^{*} U_{\tau 3} \sin ^{2} \pi \frac{\Delta m_{32}^{2}}{\Delta m_{21}^{2}}\right\}+2 \operatorname{Im}\left\{U_{e 2} U_{\tau 2}^{*} U_{e 3}^{*} U_{\tau 3} \sin 2 \pi \frac{\Delta m_{32}^{2}}{\Delta m_{21}^{2}}\right\} \\
& -4\left|U_{e 1}\right|^{2}\left|U_{e 2}\right|^{2} \sin ^{2} \pi \frac{\Delta m_{21}^{2}}{\Delta m_{21}^{2}}-4\left|U_{e 1}\right|^{2}\left|U_{e 3}\right|^{2} \sin ^{2} \pi \frac{\Delta m_{31}^{2}}{\Delta m_{21}^{2}}-4\left|U_{e 2}\right|^{2}\left|U_{e 3}\right|^{2} \sin ^{2} \pi \frac{\Delta m_{32}^{2}}{\Delta m_{21}^{2}}=0
\end{aligned}
$$

Where 


$$
\begin{gathered}
\sin \left(\pi \frac{\Delta m_{13}^{2}}{\Delta m_{21}^{2}}\right)=\sin \left(\pi \frac{24500}{750}\right)=+\frac{\sqrt{3}}{2} \\
\sin \left(\pi \frac{\Delta m_{23}^{2}}{\Delta m_{21}^{2}}\right)=\sin \left(\pi \frac{25250}{750}\right)=-\frac{\sqrt{3}}{2} \\
W=\sin ^{2}\left(\pi \frac{\Delta m_{13}^{2}}{\Delta m_{21}^{2}}\right)=\frac{3}{4} \\
W=\sin ^{2}\left(\pi \frac{\Delta m_{23}^{2}}{\Delta m_{21}^{2}}\right)=\frac{3}{4} \\
V=\sin \left(2 \pi \frac{\Delta m_{13}^{2}}{\Delta m_{21}^{2}}\right)=\sin \left(2 \pi \frac{24500}{750}\right)=-\frac{\sqrt{3}}{2} \\
V=\sin \left(2 \pi \frac{\Delta m_{23}^{2}}{\Delta m_{21}^{2}}\right)=\sin \left(2 \pi \frac{25250}{750}\right)=-\frac{\sqrt{3}}{2}
\end{gathered}
$$

Since

$$
V=\sin \left(2 \pi \frac{\Delta m_{13}^{2}}{\Delta m_{21}^{2}}\right)=\sin \left(2 \pi \frac{\Delta m_{23}^{2}}{\Delta m_{21}^{2}}\right), W=\sin ^{2}\left(\pi \frac{\Delta m_{13}^{2}}{\Delta m_{21}^{2}}\right)=\sin ^{2}\left(\pi \frac{\Delta m_{23}^{2}}{\Delta m_{21}^{2}}\right)=\frac{3}{4}
$$

The equation (29) is reduced to the form:

$$
\begin{aligned}
& 3 J\left[U_{e 1}\left(A U_{\mu 3}-E U_{\tau 3}\right)-U_{e 2}\left(C U_{\mu 3}-G U_{\tau 3}\right)\right] \cos \delta_{C P} \\
& +2 V J\left[U_{e 1}\left(U_{\tau 3} E-U_{\mu 3} A\right)+U_{e 2}\left(U_{\mu 3} C-U_{\tau 3} G\right)\right] \sin \delta_{C P} \\
& -3 U_{e 1}^{2} J^{2}-3 U_{e 2}^{2} J^{2}+3 J\left(B U_{\mu 3}+F U_{\tau 3}\right)+3 J\left(D U_{\mu 3}+H U_{\tau 3}\right) \\
& =\left(3 J \cos \delta_{C P}-2 V J \sin \delta_{C P}\right)\left(U_{e 1} A U_{\mu 3}-U_{e 1} U_{\tau 3} E+U_{e 2} C U_{\mu 3}-U_{e 2} G U_{\tau 3}\right) \\
& -3 U_{e 1}^{2} J^{2}-3 U_{e 2}^{2} J^{2}+3 J U_{e 1}\left(B U_{\mu 3}+F U_{\tau 3}\right)+3 J U_{e 2}\left(D U_{\mu 3}+H U_{\tau 3}\right)=0
\end{aligned}
$$

Or in a simplified form

$$
\left(3 J \cos \delta_{C P}-2 V J \sin \delta_{C P}\right) \varsigma-\xi=0
$$

In this equation, the following expressions equal zero:

$$
\varsigma=\left(U_{e 1} A U_{\mu 3}-U_{e 1} E U_{\tau 3}-U_{e 2} C U_{\mu 3}+U_{e 2} G U_{\tau 3}\right)=0
$$

Because

$$
\begin{gathered}
U_{\mu 3} A-U_{\tau 3} E=S_{23} C_{13} \times S_{12} C_{23}-C_{23} C_{13} \times S_{12} S_{23}=0 \\
U_{\tau 3} G-U_{\mu 3} C=C_{23} C_{13} \times C_{12} S_{23}-S_{23} C_{13} \times C_{12} S_{23}=0 \\
\xi=-3 U_{e 1}^{2} J^{2}-3 U_{e 2}^{2} J^{2}+3 U_{e 1} J\left(B U_{\mu 3}+F U_{\tau 3}\right)+3 U_{e 2} J\left(D U_{\mu 3}+H U_{\tau 3}\right)=0
\end{gathered}
$$

Because

$$
\begin{aligned}
& U_{\mu 3} B+U_{\tau 3} F-U_{e 1} J=S_{23} C_{13} \times C_{12} S_{23} S_{13}+C_{23} C_{13} \times C_{12} C_{23} S_{13}-C_{12} C_{13} S_{13}=0 \\
& U_{\mu 3} D+U_{\tau 3} H-U_{e 2} J=S_{23} C_{13} \times S_{12} S_{23} S_{13}+C_{23} C_{13} \times S_{12} C_{23} S_{13}-S_{12} C_{13} S_{13}=0
\end{aligned}
$$


Additionally, when the appropriate data from the experimental measurements are included [10], the equation (38) is reduced to the form:

$$
\left(3 \cos \delta_{C P}-2 V \sin \delta_{C P}\right) * 0=0
$$

The first point that can be stated is that this equation is always satisfied for any value of $\delta_{C P} \in[0,2 \pi)$, so such solutions make no physical sense. It is apparent that among those solutions in the range $\delta_{C P} \in[0,2 \pi)$ there is the right unique solution for the value $\delta_{C P}$. From such set of countless values, the real and unique value for $\delta_{C P}$ is drawn

from the set $\delta_{C P} \in[0,2 \pi)$ by solving the equation

$$
3 \cos \delta_{C P}-2 V \sin \delta_{C P}=0
$$

The solution of this equation presents the particular solution of the equation (45), and it is in the following form:

$$
\operatorname{tg} \delta_{C P}=\frac{3}{2 V}=\frac{3}{2 \sin \left(2 \pi \frac{\Delta m_{13}^{2}}{\Delta m_{21}^{2}}\right)}=\frac{3}{2 \sin \left(2 \pi \frac{\Delta m_{23}^{2}}{\Delta m_{21}^{2}}\right)}=\frac{3}{2\left(-\frac{\sqrt{3}}{2}\right)}=-\sqrt{3}
$$

It is especially significant to emphasize the existence of the common factor (39) in the equation (38) and it equals zero. That is why the equation could be written in the form (45). However, as we have seen, the structure of the equation for normal mass ordering (NMO) had a different form:

$$
\begin{aligned}
& \left(4 J \cos \delta_{C P} * W-2 V J \sin \delta_{C P} * V\right) * 0 \\
& =\left(4 J \cos \delta_{C P} * 0-2 V J \sin \delta_{C P} * 0\right) * 0=0 ; W \neq V ; W=0, V=0 .
\end{aligned}
$$

And, it, as such, has no sense, because the members in parentheses also equal zero. Using the procedure for calculating total probability, both for normal mass ordering (NMO) and inverted mass ordering (IMO), the same results are obtained. Namely, the total probability for normal mass ordering (23)

$$
P\left(v_{e} \rightarrow v_{\mu}\right)+P\left(v_{e} \rightarrow v_{\tau}\right)+P\left(v_{e} \rightarrow v_{e}\right)=1
$$

In addition, by equating the equation (45) with zero, the total probability in the case of inverted mass hierarchy (28) becomes

$$
P\left(v_{e} \rightarrow v_{\mu}\right)+P\left(v_{e} \rightarrow v_{\tau}\right)+P\left(v_{e} \rightarrow v_{e}\right)=1
$$

The first point that we have established is that neutrinos belong to the inverted mass hierarchy. Secondly, for that hierarchy, we can calculate the numerical value, on the basis of (47), for the Dirac CP violation phase

$$
\delta_{C P}=\operatorname{arctg}(-\sqrt{3})=-60^{0}=+300^{0}
$$

The obtained formula for the Dirac CP violation phase (47) exclusively depends on the mass splittings between the neutrino mass eigenstates in a manner established by the experiments [1-4] so that neutrino oscillations depend only on the mass splittings between the neutrino mass eigenstates.

\section{Some Properties of the Parameters of the Dirac CP Violation Phase}

If we go back to the relations provided in the formulae (47), it can be seen that the formula for the Dirac CP violation phase can be expressed through the equivalent formula

$$
\operatorname{tg} \delta_{C P}=\frac{3}{2 V}=\frac{3}{2 \sin \varphi_{31}}=\frac{3}{2 \sin \varphi_{32}}
$$

It can also be written that

$$
\operatorname{tg} \delta_{C P}=\sin \varphi_{31}+\sin \varphi_{32}
$$

As well as

$$
\operatorname{tg} \delta_{C P}=\frac{\sin \delta_{C P}}{\cos \delta_{C P}}=\frac{3}{2 V}=\frac{3}{2 \sin \delta_{C P}}
$$

And, from here

$$
\cos \delta_{C P}=\frac{2}{3} \sin ^{2} \delta_{C P}=\frac{1}{2}
$$

Returning to equations (16) and (17), it can be written:

$$
\varphi_{32}-\varphi_{31}=2 \pi \frac{\Delta m_{23}^{2}}{\Delta m_{21}^{2}}-2 \pi \frac{\Delta m_{13}^{2}}{\Delta m_{21}^{2}}=2 \pi
$$

$$
\begin{aligned}
& \sin \varphi_{32}-\sin \varphi_{31}=0 \\
& \sin \delta_{C P}=\frac{1}{2}\left(\sin \varphi_{32}+\sin \varphi_{31}\right)=\sin \varphi_{32}=\sin \varphi_{31}
\end{aligned}
$$




$$
\begin{aligned}
& \sin \left(\pi \frac{\Delta m_{23}^{2}}{\Delta m_{21}^{2}}\right)=\sin \left(\pi \frac{25250}{750}\right)=-\frac{\sqrt{3}}{2}, \cos \left(\pi \frac{\Delta m_{23}^{2}}{\Delta m_{21}^{2}}\right)=\cos \left(\pi \frac{25250}{750}\right)=+\frac{1}{2}, \\
& \sin \left(2 \pi \frac{\Delta m_{23}^{2}}{\Delta m_{21}^{2}}\right)=\sin \left(2 \pi \frac{25250}{750}\right)=2 \sin \left(\pi \frac{25250}{750}\right) \cos \left(\pi \frac{25250}{750}\right)=-\frac{\sqrt{3}}{2} \\
& \cos \left(2 \pi \frac{\Delta m_{23}^{2}}{\Delta m_{21}^{2}}\right)=\cos \left(2 \pi \frac{25250}{750}\right)=\cos ^{2}\left(\pi \frac{25250}{750}\right)-\sin ^{2}\left(\pi \frac{25250}{750}\right)=-\frac{1}{2} \\
& \sin \left(\pi \frac{\Delta m_{13}^{2}}{\Delta m_{21}^{2}}\right)=\sin \left(\pi \frac{24500}{750}\right)=+\frac{\sqrt{3}}{2}, \cos \left(\pi \frac{\Delta m_{13}^{2}}{\Delta m_{21}^{2}}\right)=\cos \left(\pi \frac{24500}{750}\right)=-\frac{1}{2} \\
& \sin \left(2 \pi \frac{\Delta m_{13}^{2}}{\Delta m_{21}^{2}}\right)=\sin \left(2 \pi \frac{24500}{750}\right)=2 \sin \left(\pi \frac{24500}{750}\right) \cos \left(\pi \frac{24500}{750}\right)=-\frac{\sqrt{3}}{2} \\
& \cos \left(2 \pi \frac{\Delta m_{13}^{2}}{\Delta m_{21}^{2}}\right)=\cos \left(2 \pi \frac{24500}{750}\right)=\cos ^{2}\left(\pi \frac{24500}{750}\right)-\sin ^{2}\left(\pi \frac{24500}{750}\right)=-\frac{1}{2} .
\end{aligned}
$$

From the relations (58) and (59), it follows:

$$
\begin{aligned}
& \sin \left(2 \pi \frac{\Delta m_{13}^{2}}{\Delta m_{21}^{2}}\right)=\sin \left(2 \pi \frac{\Delta m_{23}^{2}}{\Delta m_{21}^{2}}\right), \sin ^{2}\left(2 \pi \frac{\Delta m_{13}^{2}}{\Delta m_{21}^{2}}\right)=\sin ^{2}\left(2 \pi \frac{\Delta m_{23}^{2}}{\Delta m_{21}^{2}}\right)=\frac{3}{4} \\
& \cos \left(2 \pi \frac{\Delta m_{13}^{2}}{\Delta m_{21}^{2}}\right)=\cos \left(2 \pi \frac{\Delta m_{23}^{2}}{\Delta m_{21}^{2}}\right), \cos ^{2}\left(2 \pi \frac{\Delta m_{13}^{2}}{\Delta m_{21}^{2}}\right)=\cos ^{2}\left(2 \pi \frac{\Delta m_{23}^{2}}{\Delta m_{21}^{2}}\right)=\frac{1}{4}
\end{aligned}
$$

Moreover, we can calculate the numerical value for Jarlskog invariant $[22,23]$, using for the Dirac CPV phase $\delta_{C P}$ numerical value (51), which is

$$
J_{C P(I O)}=J_{C P(I O)}^{\max } \sin \delta_{C P}=s_{12} c_{12} s_{23} c_{23} s_{13} c_{13}^{2} \sin \delta_{C P} \approx-0.029
$$

And this value is in the vicinity of the point minimum in the Ref. NuFIT 5.0 (2020), on the CP violation graph: Jarlskog invariant, as it is depicted in Figure 1.

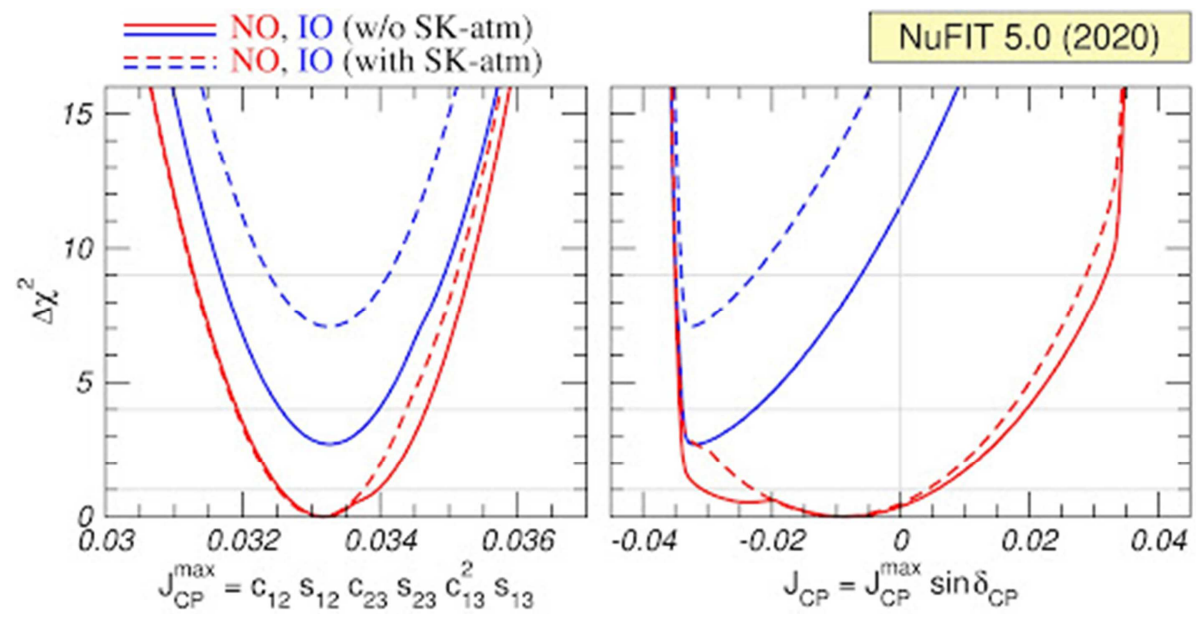

Figure 1. CP-violation: Jarlskog invariant.

\section{The Results Discussion}

The introduced theoretical research is based on the latest data presented in the Ref. [10]. The selection of data processed in that manner is given in the Ref. [10], depicting neutrino oscillation parameters summary determined from the global analysis. Neutrino oscillations depend only on the mass splittings between the neutrino mass eigenstates. From the Ref. [10] parameters for both types of ordering are selected: 
Normal ordering

$$
\begin{gathered}
\theta_{12}=34.3 \pm 1^{0}, \theta_{23}=49.26 \pm 0.79^{0}, \theta_{13}=8.53_{-0.53}^{+0.130}, c_{i j}=\cos \theta_{i j}, s_{i j}=\sin \theta_{i j} ; i, j=1,2,3 . \\
\Delta m_{21}^{2}=7.50_{-0.20}^{+0.22} \times 10^{-5} \mathrm{eV}^{2}, \Delta m_{31}^{2}=2.55_{-0.03}^{+0.02} \times 10^{-3} \mathrm{eV}^{2}
\end{gathered}
$$

Inverted ordering

$$
\begin{gathered}
\theta_{12}=34.3 \pm 1^{0}, \theta_{23}=49.46 \pm 0.79^{0}, \theta_{13}=8.58_{-0.53}^{+0.130}, c_{i j}=\cos \theta_{i j}, s_{i j}=\sin \theta_{i j} ; i, j=1,2,3 . \\
\Delta m_{21}^{2}=7.50_{-0.20}^{+0.22} \times 10^{-5} \mathrm{eV}^{2}, \Delta m_{13}^{2}=2.45_{-0.03}^{+0.02} \times 10^{-3} \mathrm{eV}^{2}
\end{gathered}
$$

Central values from the best fit $\pm 1 \sigma$ are taken for calculations:

Normal ordering

$$
\begin{gathered}
\theta_{12}=34.3^{0}, \theta_{23}=49.26^{0}, \theta_{13}=8.53^{0}, c_{i j}=\cos \theta_{i j}, s_{i j}=\sin \theta_{i j} ; i, j=1,2,3 . \\
\Delta m_{21}^{2}=7.50 \times 10^{-5} \mathrm{eV}^{2}, \Delta m_{31}^{2}=2.55 \times 10^{-3} \mathrm{eV}^{2}, \Delta m_{32}^{2}=2.475 \times 10^{-3} \mathrm{eV}^{2}
\end{gathered}
$$

Inverted ordering

$$
\begin{aligned}
& \theta_{12}=34.3^{0}, \theta_{23}=49.46^{0}, \theta_{13}=8.58^{0}, c_{i j}=\cos \theta_{i j}, s_{i j}=\sin \theta_{i j} ; i, j=1,2,3 \\
& \Delta m_{21}^{2}=7.50 \times 10^{-5} \mathrm{eV}^{2}, \Delta m_{13}^{2}=2.45 \times 10^{-3} \mathrm{eV}^{2}, \Delta m_{23}^{2}=2.525 \times 10^{-3} \mathrm{eV}^{2}
\end{aligned}
$$

Applying the adopted values for the parameters (64) and (65), we obtained the results (21) and (25) on the basis of which normal mass ordering is disfavoured with no restrictions.

On the basis of the motion equation (29), we obtained the final equation for the Dirac CP violation phase (45), the global solution of which is that there are countless solutions for $\delta_{C P}$, which has no physical sense and must be rejected. However, the equation (46) offers a particular solution for $\delta_{C P}$ and it is essentially singled out from countless solutions offered by the equation (45), but being singled out, it represents a possible solution that makes physical sense for the Dirac CP violation phase (47).

The main characteristic of the solution for CP phase presented by the equation (47) is that it exclusively depends on the mass splittings between the neutrino mass eigenstates.

For the sake of comparison, both obtained numerical values, for CP phase (47) and $\delta_{C P} / \pi \approx 1.667$, are in the vicinity of the local minimum of the inverted ordering, as depicted by the graphs given in the Ref.[10].

\section{Conclusion}

The purpose of this paper was to obtain an explicit value of the Dirac CP violation phase. Two examples were analyzed: The first example represents normal mass ordering, which is unconditionally excluded due to the structure of equation (25), and which as such does not offer any solution for the CP phase. Furthermore, when it comes to the structure of the neutrino mass hierarchy, it can be determined on the basis of the formula (14). Our research is based on the data obtained on the basis of processing the latest experimental measurements exhibited in the Ref. [10].

On the basis of the complex structure of the motion equation (29), we obtained the final simple equation for the Dirac CP violation phase (45) whose particular solution for $\delta_{C P}$ has physical sense and it is given in the formula (47).

It is especially emphasized that the main feature of this solution is that it depends exclusively on the mass splittings between the neutrino mass eigenstates, which is also the main conclusion regarding the existence of the phenomenon of neutrino oscillations based on the mass splittings between neutrino mass eigenstates as shown by the experiments [1-5]. This solution differs from other solutions given in Refs. [13, 15, 16-19]

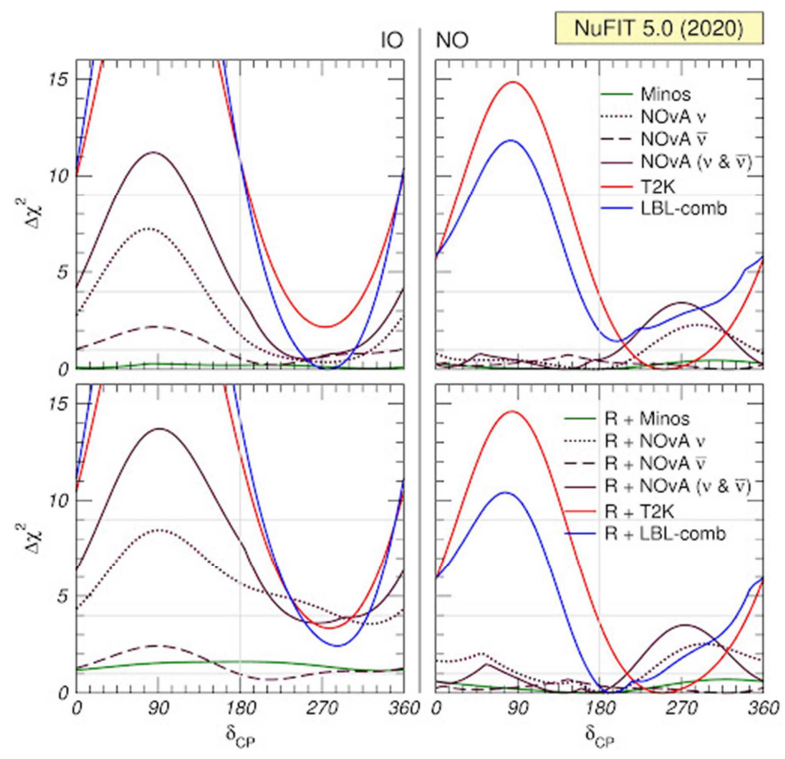

Figure 2. Determination of $\delta_{C P}$. 
The graphs shown in Refs. $[10,12]$ clearly depict that the obtained numerical values, for $\mathrm{CP}$ phase (47) and $\delta_{C P} / \pi \approx 1.667$, are in the vicinity of the local minimum of the inverted ordering, as it is depicted in Figure 2.

Checking the existence of the inverted mass hierarchy could also be easily established through the relation (14). The theoretical results presented in this paper are exclusively related to the data in Ref. [10]. However, if the published data [10] change, that could influence the outcome of the final derived results. Moreover, it is particularly emphasized that there are opinions that neutrino mass hierarchy could have a normal structure [11], but there is no clear and convincing argumentation for that. Additionally, there is an opinion that given result in the Ref. [14] does not provide a strong evidence of normal hierarchy over inverted hierarchy. That opinion could be checked through comparison of wavelengths of oscillations given in the formula (5).

Finding a numerical value of the Dirac CP violation phase (51) would enable the calculation of the three neutrino mass eigenstates, as well as the possible effective value of the neutrino Majorana mass, which could appear in the process of the neutrinoless double beta decay $[20,21]$.

\section{References}

[1] K. Nakamura et al (2010), "Review of particle physics", Journal of Physics G Nucl. Part. Phys. 37075021.

[2] M. Tanabashi et al., Particle data group, Review of particle physics, Phys. Rev. D 98, 030001 (2018).

[3] Fukuda Y. Hajokawa, T. Ichichara, E., Inove K., Ishicara K., IshinoK., et al. (Super-Kamiokande Collaboration) (1998) Evidence for oscillation of atmospheric neutrinos. Phys. Rev. Lett. 81 1562-1567.

[4] Abe, K. et al. T2K Collaboration (August 2013), Evidence of electron neutrino appearance in a muon neutrino beam, Physical Review D 88 (3) 032002 arxiv.1304.0841.

[5] Particle Data Group Collaboration, Review of particle physics, Phys. Rev. D 98 (2018) 3, 030001.

[6] M. A. Acero et al., New constraint on oscillation parameters from $v_{e}$ appearance and $v_{\mu}$ disappearance in the NOvA experiment, Phys. Rev. D98, 032012 (2018).

[7] K. Abe et al. [T2K], Search for CP violation in neutrino and antineutrino oscillations by the T2K experiment with $2.2 \times 10^{21}$ protons on target, Phys. Rev. Let. 121, 187802 (2018).

[8] C. Giganti, S. Levignac, M. Zito, Neutrino oscillations: the rise of the PMNS paradigm, arxiv: $1710.00715 \mathrm{v} 2$ [hep-ex] 16 Nov 2017.

[9] R. B. Patterson, Prospect measurement of the neutrino mass hierarchy, Annual Review of Nuclear and Particle Science, Volume 65, 2015/Patterson/pp. 177-192.

[10] P. F. de Salas, D. V. Forero, S. Gariazzo, P. Maetinez-Mirave, O. Mena, C. A. Termes, M. Tortola and J. W. F. Valle, 2020 global reassessment of the neutrino oscillations picture, JHEP 02 (2021) 071.

[11] P. F. de Salas, S. Garazzo, O. Mena, C. A. Ternes and M. Tortola, Neutrino mass ordering from oscillations and beyond: 2018 status and future prospects, Front. Astron. Space Sci. 09 October 2018.

[12] NuFIT 5.0 (2020).

[13] Planck 2018 results: VI. Cosmological parameters, arxiv: 1807.06209v2 [astro-ph.CO] 20 Sep 2019.

[14] S. R. Choudhury, S. Hannestad, Updated results on neutrino mass and mass hierarchy from cosmology with Planck 2018 likelihood, arxiv: 1907.12598v3, 15 Jul 2020.

[15] I. Esteban, M. C. Gonzale-Garcia, A. Hernandez-Cabezudo, M Maltoni, T. Schwetz, Global analysis of three flavor neutrino oscillations: synergies and tensions in the determinations of $\theta_{23}, \delta_{c p}$, and the mass ordering, J. High Energy Phys. 01 (2019) 106.

[16] I. Girardi, S. T. Petcov, A. V. Titov, Determining the Dirac CP violation phase in the neutrino mixing matrix from sum rules, Nucl. Phys. B 894 (2015) 733-768.

[17] H. Okada and M. Tanimoto, Spontaneous CP violation by modulus $\tau$ in A4 model of lepton flavours, JHEP 03 (2021) 010 .

[18] J. Gehrlein and M. Spinrath, Highlight the correlation between the Dirac CP V phase and the atmospheric mixing angle to the allowed range for the neutrino mass scale, JHEP 02 (2021).

[19] G. Ghosh, Significance of Broken $\mu-\tau$ Symmetry in Correlating $\delta_{C P}, \theta_{13}$, Lightest Neutrino Mass, and Neutrinoless Double Beta Decay 0v $\beta \beta$., Advances in High Energy Physics, Volume 2021, Article ID 956 3917, 23 page.

[20] L. Graf, S. Jana, M. Lindner, W. Rodejohann, and Xun-jie Xu, Flavored neutrinoless double beta decay, Physical Review D 103, 055007 (2021).

[21] A. Formaggio, A. L. C. de Gouvea, R. G. H. Robertson, Direct measurements of neutrino mass, arxiv: $2102.00594 \mathrm{v} 2$ [nuclexp] $11 \mathrm{Feb} 2021$.

[22] H. Georgi and C. Jarlskog, Phys. Lett. 86B, 297, (1979).

[23] C. Jarlskog, Phys. Rev. Lett. 55, 1039 (1985). 